Transcriptional Activation by SP1 as Directed Through TATA or Initiator: Specific Requirement for Mammalian Transcription Factor IID

ST Smale, MC Schmidt, AJ Berk, and D Baltimore

PNAS 1990;87;4509-4513

doi:10.1073/pnas.87.12.4509

This information is current as of December 2006.

E-mail Alerts

Rights \& Permissions

Reprints
This article has been cited by other articles:

www.pnas.org\#otherarticles

Receive free email alerts when new articles cite this article - sign up in the box at the top right corner of the article or click here.

To reproduce this article in part (figures, tables) or in entirety, see: www.pnas.org/misc/rightperm.shtml

To order reprints, see:

www.pnas.org/misc/reprints.shtml

Notes: 


\title{
Transcriptional activation by Sp1 as directed through TATA or initiator: Specific requirement for mammalian transcription factor IID
}

\author{
(yeast transcription factor IID/RNA polymerase II/transcription initiation)
}

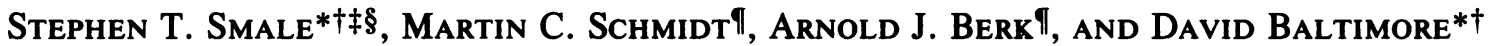 \\ *Whitehead Institute for Biomedical Research, 9 Cambridge Center, Cambridge, MA 02142; ' Department of Biology, Massachusetts Institute of Technology, \\ Cambridge, MA 02139; and "Molecular Biology Institute and Department of Microbiology, University of California, Los Angeles, CA 90024
}

\begin{abstract}
Transcription of mammalian genes by RNA polymerase II often begins at a specific nucleotide, whose location is determined either by an upstream DNA element known as a TATA box or by an element positioned at the transcription start site called an initiator (Inr). By in vitro analysis of synthetic promoters, we demonstrate here that the TATA and Inr elements are functionally similar and that the Inr is contained between nucleotides -3 and +5 relative to the initiation site. Moreover, we found that a mammalian transcription factor IID (TFIID) protein fraction is required for transcriptional stimulation by an Sp1-dependent activating element placed upstream of either TATA or Inr elements. However, in these assays, the yeast TATA-binding protein, which previously was shown to function similarly to mammalian TFID, could not efficiently substitute for the mammalian TFIID fraction. These results demonstrate that mammalian TFIID is functionally distinct from the yeast TATA-binding protein and may contain additional subunits or domains that are important for transcriptional activation from some promoters.
\end{abstract}

The regulated transcription of a typical eukaryotic gene is governed by the combined action of multiple sequencespecific DNA-binding proteins (1). The information provided by these proteins is ultimately communicated to RNA polymerase II, resulting in a precise transcription initiation frequency. In many genes, a TATA box positions the transcription start site to a point 30 nucleotides downstream (2), suggesting that this element plays an important role in relaying information to RNA polymerase from factors bound to distal control elements. A chromatographic fraction termed transcription factor IID (TFIID) that stimulates transcription initiation $(3,4)$ and contains a DNA-binding activity that specifically interacts with TATA sequences (5) has been derived from mammalian (6) and Drosophila (7) cells. A protein with similar activities has been identified in yeast (8) and the gene encoding this yeast TATA-binding protein (referred to as yeast TFIID) has been isolated (9-13).

Recently, it was shown that no TATA elements are found in either the small nuclear RNA (snRNA) genes or the murine terminal deoxynucleotidyltransferase (TdT) gene, but that distinct elements determine the precise transcription start sites. For snRNA genes, an element located $\approx 60$ nucleotides from the start site is crucial for promoter function and start site placement (14). TdT transcription requires an initiator (Inr) element (15-17) that directly overlaps the start site and exhibits sequence homology to start-site regions for other polymerase II-transcribed genes. In vitro experiments ascribed two distinct activities to an Inr (15): (i) the ability to

The publication costs of this article were defrayed in part by page charge payment. This article must therefore be hereby marked "advertisement" in accordance with 18 U.S.C. $\$ 1734$ solely to indicate this fact. independently direct RNA polymerase II to initiate transcription from a specific, internal position; and (ii) the ability to be activated in the absence of TATA by an upstream activator element, resulting in high levels of accurate transcription.

In this paper, we describe further characterization of an Inr, beginning with a direct comparison of the effects of Inr and TATA on the functioning of a transcription factor Sp1dependent upstream activator element. We also describe the activities of a series of Inr mutants and have begun to address the protein requirements for Inr-mediated transcription.

\section{MATERIALS AND METHODS}

Plasmid DNAs. The plasmids designated I-VII (see Fig. 1 Lower) are similar to plasmids described previously (15). They contain oligonucleotides for the TdT Inr or the adenovirus major late (AdML) promoter TATA box, or a restriction fragment containing the simian virus 40 (SV40) 21base-pair (bp) repeats (15), inserted into the pSP72 (Promega) polylinker. Plasmid I was called TdT17mer(pSP72), plasmid II contains the TATA oligonucleotide in the pSP72 EcoRI and Sac I sites, plasmid III contains the 21-bp repeats in the pSP72 Bgl II site as in the previously described pSp1 42 Inr (but without the Inr oligonucleotide), plasmid IV was called TATA/17mer(pSP72), plasmid V contains the 21-bp repeats as in plasmid III plus the TATA oligonucleotide as in plasmid II, plasmid VI was called pSP1 42 Inr, and plasmid VII contains the TATA oligonucleotide in the EcoRI and Sac I sites of pSP1 42 Inr.

Plasmids containing Inr mutations were constructed with synthetic oligonucleotides, which were inserted into the $S a c$ I and BamHI sites of pUC18 for Inr-only transcription, and into plasmid III for Sp1-Inr transcription. All plasmids were purified twice on $\mathrm{CsCl}$ equilibrium gradients (18).

In Vitro Transcription. Nuclear extracts were prepared from HeLa cells as described $(15,19,20)$. Selective heat inactivation of TFIID at $47^{\circ} \mathrm{C}$ was by the method of Nakajima et al. (6). Nuclear extracts were depleted of TFIID by chromatography on phosphocellulose (21).

HeLa TFIID ( $0.6 \mathrm{mg}$ of protein per $\mathrm{ml}$ ) was prepared by chromatography on phosphocellulose and DEAE-cellulose as described (21). The FPLC Mono S column was prepared with a nuclear extract from RLm11 T cells that had first been applied to a heparin-agarose column and eluted with a 0.2-0.4 M KCl step. This fraction (4 mg) was loaded onto a $1-\mathrm{ml}$ Mono $S$ column in $25 \mathrm{mM}$ Hepes, $\mathrm{pH} 7.9 / 10 \%$ (vol/vol)

Abbreviations: Inr, initiator; TFIID, transcription factor IID; TdT, terminal deoxynucleotidyltransferase; AdML, adenovirus major late; SV40, simian virus $\mathbf{4 0}$.

$\ddagger$ Present address: Howard Hughes Medical Institute and Department of Microbiology and Immunology, University of California, Los Angeles, School of Medicine, Los Angeles, CA 90024

$\S$ To whom reprint requests should be addressed. 
glycerol/0.2 mM EDTA/1 mM dithiothreitol/100 mM KCl. The column was washed with $5 \mathrm{ml}$ of loading buffer and eluted at $0.4 \mathrm{ml} / \mathrm{min}$ with a $20-\mathrm{ml}$ linear $\mathrm{KCl}$ gradient (0.1-0.5 M). One-milliliter fractions were collected.

Yeast TFIID was purified (21) or overexpressed in Escherichia coli prepared as described (11).

In vitro transcription reactions were performed as described $(15,22)$, with $10 \mu$ l of crude or heat-inactivated extract $(20 \mathrm{mg} / \mathrm{ml})$ containing appropriate fractions. Primer extension was performed as described $(15,23)$. We confirmed all results from plasmid I with $\mathrm{S} 1$ nuclease analysis (data not shown; see ref. 15). The signals from S1 nuclease experiments are extremely weak, but the results are more consistent and eliminate difficulties arising from incomplete reverse transcription.

\section{RESULTS}

Comparison of TATA and Inr. We inserted into a plasmid vector, in various combinations, oligonucleotides containing TATA or Inr elements, as well as in some constructs a restriction fragment containing an upstream activator element, the SV40 21-bp repeats [containing six binding sites for transcription factor Sp1 (24)]. The plasmids, labeled with roman numerals in Fig. 1, were tested for promoter activity by in vitro transcription and primer-extension analysis. Consistent with previous findings by us $(15)$ or others $(2,25,26)$, plasmids containing the isolated Inr (plasmid I), TATA

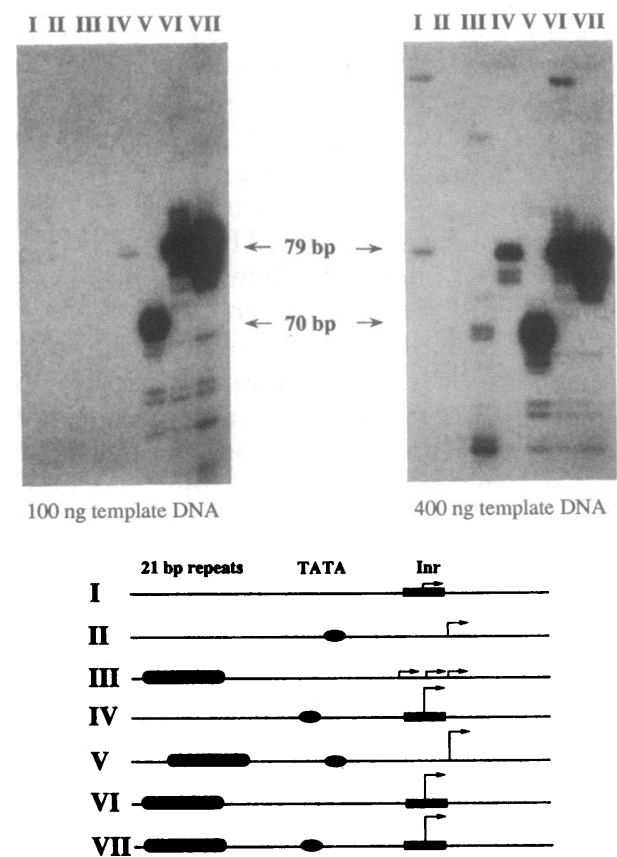

Fig. 1. In vitro transcription from synthetic promoters. Oligonucleotides containing either the AdML TATA or TdT Inr, or a fragment containing the SV40 21-bp repeats, were inserted into plasmid pSP72 (see Materials and Methods and ref. 15). Distances between the 21-bp repeats and TATA or Inr were varied to obtain maximal signals (15). The distance between TATA and Inr is $27 \mathrm{bp}$, the distance between the 21-bp repeats and Inr is $42 \mathrm{bp}$, and the distance between the 21-bp repeats and TATA is $30 \mathrm{bp}$. In vitro transcription reactions were performed in HeLa nuclear extracts with $100 \mathrm{ng}$ (Upper Left) or $\mathbf{4 0 0} \mathrm{ng}$ (Upper Right) of supercoiled template. RNA was analyzed by primer extension with a SP6 promoter primer (Promega) and electrophoresed on an $8 \%$ denaturing polyacrylamide gel. The dried gel was exposed for $2 \mathrm{hr}$ with an intensifying screen. Sizes of extension products were 79 nucleotides (lanes I, IV, VI, and VII) or 70 nucleotides (lanes II and V), as indicated. The promoters used for each reaction are diagrammed at the bottom. (plasmid II), or upstream activator (plasmid III) promoted transcription initiation, but at a very low frequency. With 400 ng of DNA (Fig. 1 Upper Right), accurate initiation was detected from within the Inr (lane I) or from heterogeneous nucleotides downstream of the 21-bp repeats (lane III). A signal was not detected from plasmid II with the exposure shown here (lane II) but was visible with longer exposures (see Fig. $3 A$, lane 1). With 100 ng of template, these signals were weaker (Fig. 1 Upper Left, lanes I, II, and III). When TATA was positioned 30 bp upstream of the Inr (plasmid IV), promoter strength increased but remained relatively weak with either 100 or $400 \mathrm{ng}$ of DNA (Fig. 1 Upper, lanes IV). To obtain high levels of transcription, the 21-bp repeats had to be positioned upstream of either TATA or Inr. With a promoter containing the 21-bp repeats and TATA (plasmid V), efficient transcription began 30 bp downstream from TATA (lanes V). This promoter strength varied by only 3-fold as the 21-bp repeats were moved from 12 to 60 bp upstream of TATA (data not shown). With a promoter containing the 21-bp repeats and Inr (plasmid VI), equally strong transcription began from within the Inr (lanes VI). The variability of promoter strength with distance between the 21-bp repeats and Inr has been reported (15). When both TATA and Inr were downstream of the 21 -bp repeats (plasmid VII), with TATA appropriately positioned $30 \mathrm{bp}$ from Inr, promoter strength increased by another 3- to 5-fold (lanes VII). These results demonstrate that either an Inr or a TATA box is sufficient to allow the 21-bp repeats to activate transcription efficiently and with site specificity.

Analysis of Inr Mutants. Mutant Inr elements were prepared and tested for two activities in nuclear extracts: $(i)$ the ability to direct transcription independently; and (ii) the ability to direct high levels of accurate transcription in the presence of the 21-bp repeats. One set of mutants contains substitutions in the central region of the Inr and are called C1, C2, C3, and C4 (Fig. 2). In crude extracts, C4 behaved like the wild-type Inr (lanes 5 and 15), but $C 1, C 2$, and $C 3$ impeded both independent Inr transcription (lanes 2-4) and Inr activation by the 21 -bp repeats (Fig. 2 , lanes $12-14$, bottom). The effects of the mutations on transcription mediated by the 21-bp repeats became even greater when the mutants were tested in a competition assay with plasmid $\mathrm{V}$, which was added to each reaction mixture in equimolar amounts (Fig. 2, lanes $12-14$, top). Interestingly, mutant $C 2$ altered the start site to the $G$ that was placed at the -1 position.

A second set of mutants, F1, F2, and F3, contained substitutions in the flanking regions of the Inr. These mutations had little effect on transcription in the absence of the 21-bp repeats (Fig. 2, lanes 7-9). However, F1 and F3, which both alter nucleotides +3 to +5 , only directed low levels of transcription when the 21-bp repeats were present (Fig. 2, lanes 18 and 20: top, with competitor; bottom, without competitor). We previously showed that a mutation at nucleotides -2 and -3 strongly diminished Inr transcription and that a $\mathrm{T}$ to $\mathrm{C}$ transition at nucleotide +2 had no effect (15). Taken together, these data show that the functional TdT Inr is contained within $\approx 8 \mathrm{bp}$, from -3 to +5 (Fig. 2 Lower).

Requirement for Mammalian TFIID. TATA-mediated transcription requires recognition of the TATA box by a DNAbinding protein, TFIID (3-7). Because we detected efficient transcription in the absence of TATA and in the presence of $\mathrm{Spl}$ and Inr sites, it was important to determine whether this transcription required TFIID. To test for the TFIID requirement, we made use of the observation that TFIID activity can be depleted selectively from HeLa nuclear extracts by mild heat treatment (6). With templates II, IV, V, or VI, transcription was abolished by this heat treatment (Fig. 3A, lanes $2,5,8$, and 11). More importantly, efficient transcription was recovered in all cases by addition of partially purified HeLa TFIID (21) (lanes 3, 6, 9, and 12) that is known to lack RNA 


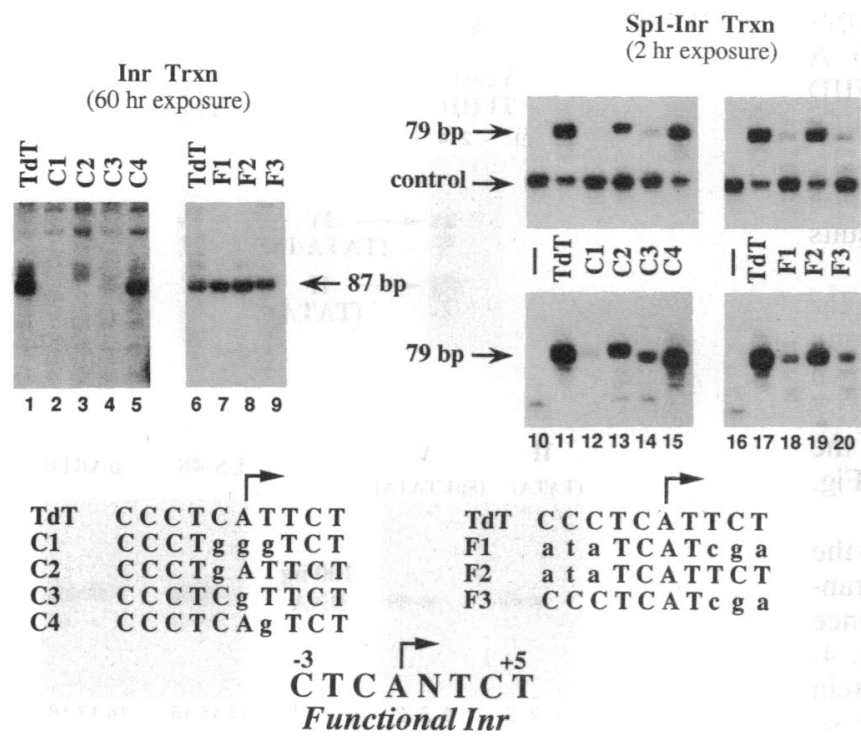

FIG. 2. In vitro transcription with Inr substitution mutants. Oligonucleotides containing substitutions in the TdT Inr, as indicated at the bottom, were inserted into pUC18 to test for independent Inr activity (lanes 1-9), or into plasmid III to test for activity in the presence of the 21-bp repeats (lanes 10-20). In vitro transcription reactions were performed with $400 \mathrm{ng}$ (lanes 1-9) or $100 \mathrm{ng}$ (lanes 10-20) of template DNA and RNA was analyzed by primer extension. The exposure for lanes $1-9$ is longer $(60 \mathrm{hr})$ than the exposure for lanes $10-20(2$ $\mathrm{hr})$. The expected products are indicated by arrows (87 bp for lanes 1-9 and 79 bp for lanes 10-20). $\mathrm{Sp} 1 /$ Inr transcription was tested in both the presence (lanes 10-20, top) and absence (lanes 10-20, bottom) of an internal control, which was plasmid V. A proposed sequence of the functional Inr is shown at the bottom. We previously showed that a double mutant at nucleotides -2 and -3 effected Inr transcription and that a $\mathrm{T}$ to $\mathrm{C}$ transition at +2 had no effect (15). Also, by comparison to the AdML promoter Inr, nucleotides +6 to +11 were unimportant (15). polymerase II, Sp1, and other general transcription factors (data not shown). This activity was recovered at a similar rate with both construct VI and V, as is apparent from a TFIID titration (Fig. 3B). Thus, HeLa TFIID fraction was required for transcriptional activation by the 21 -bp repeats, regardless of whether the transcription was mediated by TATA or Inr.

To address the possibility that HeLa TFIID contains two distinct but heat-sensitive activities, one required for Inr transcription and one for TATA transcription, we examined the elution profiles of the stimulatory activities from a high-resolution FPLC Mono S cation-exchange column. The stimulatory activities for both plasmids V and VI exactly coeluted from a $\mathrm{KCl}$ gradient on the Mono S column (Fig. $3 B$; the stimulatory activities for plasmids II and IV also coeluted; data not shown). These experiments strongly suggest that the same protein or protein complex previously defined as TFIID is required for $\mathrm{Sp} 1$ to activate transcription from either a TATA box or an Inr element.

A similar requirement for HeLa TFIID was found with the weak heterogeneous transcription seen with plasmid III (data not shown). We do not know, however, whether this transcription is mediated by several weak TATA and Inr ele- ments that may be fortuitously located downstream from the 21-bp repeats. We have not reached a conclusion concerning a TFIID requirement for plasmid I transcription. By S1 nuclease analysis, heat-inactivation of the HeLa extracts eliminated transcription (data not shown). However, addition of TFIID did not recover the activity to detectable levels, even though full recovery was observed with TATAcontaining constructs by $\mathrm{S} 1$ nuclease analysis (data not shown). Among other possibilities, this result may suggest that TFIID is not required for plasmid I transcription, but that heat inactivation eliminates transcription in this case by partially inactivating another protein (e.g., RNA polymerase or TFIID) that is required for transcription, but that is not limiting in the presence of TATA or an upstream activator.

The Activity of the Yeast TATA-Binding Protein. Recently, a TATA-binding protein from yeast was purified (8) and cloned (9-13) and was shown to substitute for mammalian TFIID in reconstituted HeLa in vitro transcription assays $(8-13,21)$. It therefore was anticipated that this yeast TFIID would be active in our experiments. Surprisingly, however, the yeast protein did not function like mammalian TFIID. The most dramatic difference was observed with construct VI, where
A
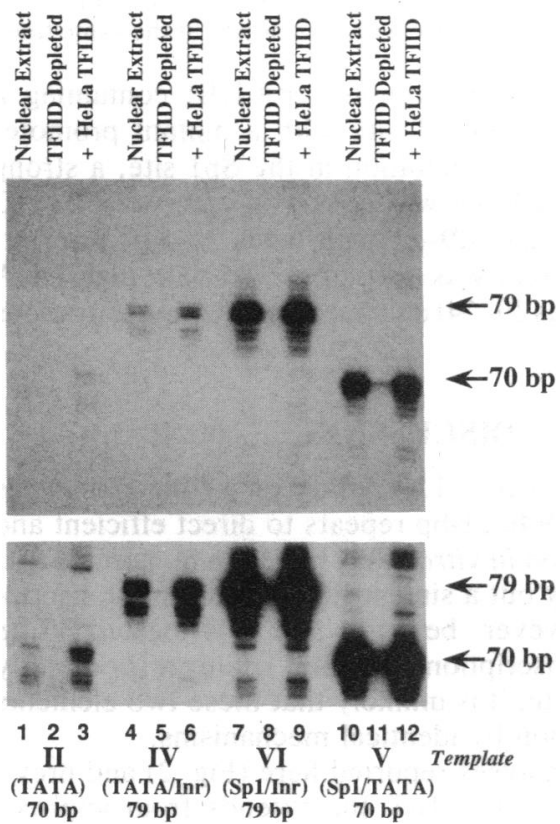

B

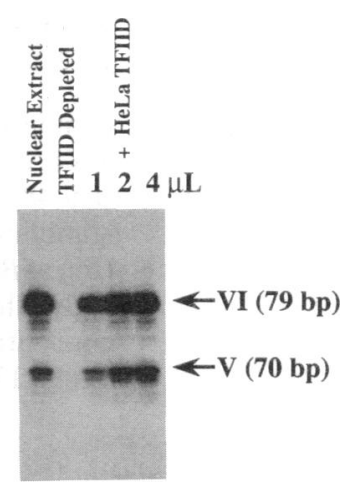

12345

C

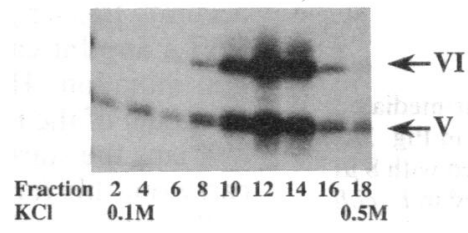

FIg. 3. HeLa TFIID requirement for TATAand Inr-mediated transcription. In vitro transcriptions were assayed by primer extension as described in Fig. 1. (A) Transcription reactions were performed with $200 \mathrm{ng}$ of plasmids II (lanes 1-3), IV (lanes 4-6), VI (lanes 7-9), and V (lanes 10-12), using $200 \mathrm{mg}$ of crude nuclear extract (lanes $1,4,7$, and 10), heat-inactivated extract (to deplete TFIID; lanes $2,5,8$, and 11), or heat-inactivated extract supplemented with $2 \mu$ l of partially purified HeLa TFIID (lanes 3, 6, 9, and 12). Short (top lanes) and long (bottom lanes) exposures of the gel are shown, and the template and expected size of extension product for that template are indicated at the bottom. The arrows indicate the locations of the 70and 79-bp products. $(B)$ One hundred nanograms of plasmids VI (79-bp extension) and V (70-bp extension) were mixed and tested as described above, using crude extract (lane 1), heat-inactivated extract (lane 2), or heat-inactivated extract with 1 (lane 3), 2 (lane 4), or 4 (lane 5) $\mu$ l of HeLa TFIID. (C) One hundred nanograms of plasmids VI and V were assayed with heat-inactivated extract supplemented with $10 \mu \mathrm{l}$ of column fraction from FPLC Mono $S$ column. Fraction number and the beginning and ending $\mathrm{KCl}$ concentration from the linear salt gradient are indicated. 
yeast TFIID expressed in $E$. coli failed to stimulate transcription in heat-inactivated HeLa extracts (Fig. 4, lanes 7-9). A similar result was found with authentic, purified yeast TFIID and with HeLa extracts depleted of mammalian TFIID by column chromatography (data not shown). This result could be due to the inability of yeast TFIID to mediate an interaction with either the 21-bp repeats or the Inr; the following results suggest that both interactions may be defective.

First, yeast TFIID was defective in its response to an Inr element. An Inr typically had a 10-fold effect on transcription directed by TATA in the presence of HeLa TFIID, as shown in Fig. 5A (Right) by comparing transcription of plasmids IV and II. By contrast, in the presence of yeast TFIID, the promoter strengths of plasmids IV and II were identical (Fig. $5 A$ Left).

Moreover, yeast TFIID was defective in its response to the SV40 21-bp repeats. Yeast TFIID strongly activated transcription from TATA-containing constructs, but the presence of the 21-bp repeats had only a 3- to 5-fold effect (Fig. 4, compare lanes 3 and 12). Even though enough yeast protein was added for efficient transcription from plasmid II (lane 3), it could not restore plasmid $\mathrm{V}$ transcription to the level seen in crude extract (Fig. 4, compare lanes 10 and 12). This result is very different from that observed in Fig. $3 A$, where much less HeLa TFIID activity (as determined with plasmid II; compare Fig. $3 A$, lane 3, to Fig. 4, lane 3) completely restored plasmid V transcription (compare Fig. 3A, lanes 10 and 12, to Fig. 4, lanes 10 and 12).

A more complete deficiency in $\mathrm{Spl}$ responsiveness was observed when authentic, purified yeast TFIID was added to extracts depleted of HeLa TFIID by phosphocellulose chromatography (Fig. 5B). In this case, addition of yeast protein with plasmids II (lanes 1-3 and 7-9) and V (lanes 4-6 and 10-12) showed no effect of the Sp1 sites. This result was observed with either 100 (lanes 1-6) or 2000 (lanes 7-12) ng of template DNA. This is in contrast to the addition of HeLa TFIID, which resulted in a 10 -fold stimulation by the 21-bp repeats (data not shown). The results also differ from experiments reported previously by two of us (21) and expanded on in Fig. 5B, with adenovirus E1B promoter plasmids. By

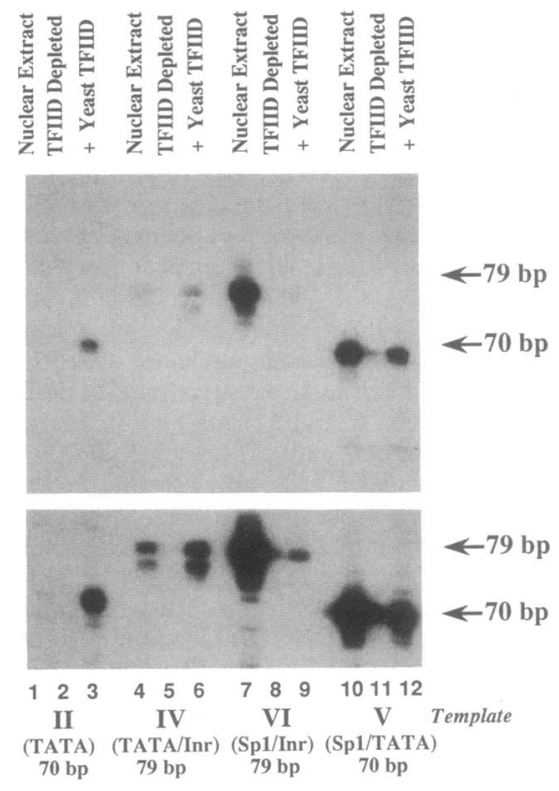

Fig. 4. Yeast TFIID requirement for TATA- and Inr-mediated transcription. This experiment was performed exactly as in Fig. $3 A$, except reactions in lanes $3,6,9$, and 12 were supplemented with $8 \mu \mathrm{l}$ of partially purified yeast TATA-binding protein expressed in $E$. coli (see Materials and Methods). Similar results were found with authentic, purified yeast TATA-binding protein (data not shown).
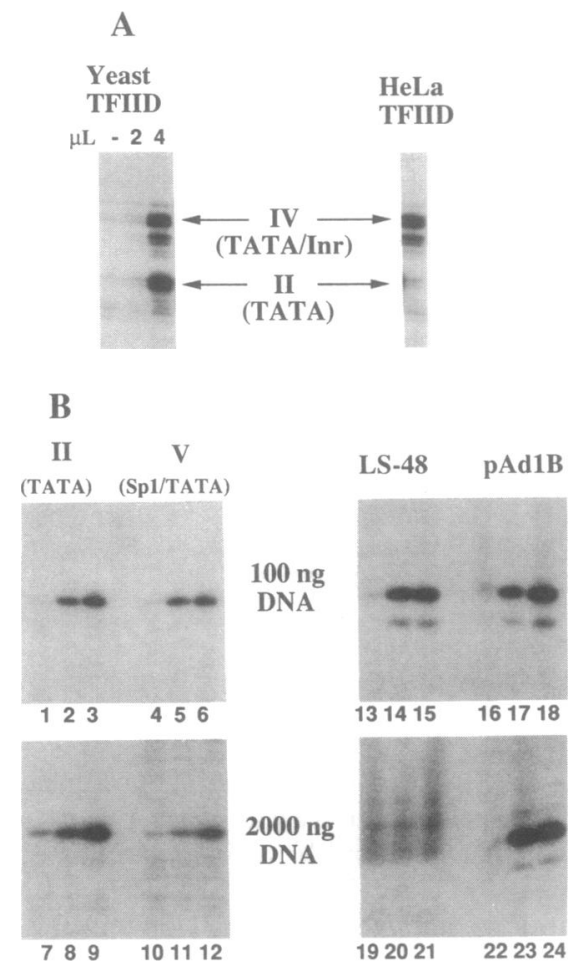

FIG. 5. Yeast TFIID activity on synthetic and adenovirus E1B promoters. $(A)$ Two hundred nanograms each of plasmids II and IV were mixed and assayed with $200 \mathrm{mg}$ of heat-inactivated extract and either 2 or $4 \mu$ l of yeast TFIID (Left) or $2 \mu$ l of HeLa TFIID (Right). Arrows indicate sizes of expected products for plasmid IV (79 bp) or plasmid II (70 bp). (B) A HeLa crude extract was depleted of TFIID by fractionation on phosphocellulose (see Materials and Methods). In vitro reactions were performed with $3 \mu$ l of $0.1-0.6 \mathrm{M} \mathrm{KCl}$ phosphocellulose step, containing 0 (lanes $1,4,7,10,13,16,19$, and 22), 1 (lanes $2,5,8,11,14,17,20$, and 23), or 4 (lanes $3,6,9,12,15$, 18,21 , and 24) $\mu \mathrm{l}$ of a $1: 5$ dilution of purified yeast TFIID. The template DNAs were either plasmid II (lanes 1-3 and 7-9) compared with plasmid V (lanes 4-6 and 10-12) or the wild-type E1B promoter plasmid pAd1B (lanes 16-18 and 22-24) compared with a mutation in the Sp1 site of the E1B promoter (plasmid LS-48/-39; lanes 13-15 and 19-21). The E1B plasmids have been described (21). Either $100 \mathrm{ng}$ (lanes 1-6 and 13-18) or 2000 ng (lanes 7-12 and 19-24) of plasmid DNA was used in each reaction. Primer extensions were performed with the SP6 primer (lanes 1-12) or primer B1750 (see ref. 21; lanes 13-24). Extension products were 70 bp (lanes 1-12) or 52 bp (lanes 13-24). All reactions in this figure were performed at the same time.

comparing the wild-type promoter (pAd1B) containing a TATA box and a single Sp1 site with a mutant promoter (LS-48/-39) containing a mutation in the Sp1 site, a strong stimulation by the $\mathrm{Sp} 1$ site was observed with yeast TFIID (Fig. 5B, compare lanes 19-21 with lanes 22-24). Interestingly, this strong effect was only observed with high DNA concentrations (lanes 19-24) and not with low concentrations (lanes 13-18).

\section{DISCUSSION}

It is evident that either a TATA box or an Inr element is necessary for the SV40 21-bp repeats to direct efficient and accurate transcription in vitro. This result demonstrates that TATA and Inr carry out a similar function during transcription initiation. However, because TATA is located $30 \mathrm{bp}$ upstream of the transcription start site, while an Inr directly overlaps the start site, it is unlikely that these two elements carry out this function by identical mechanisms.

The mutational analyses reported here (Fig. 2) and previously (15) reveal that the TdT Inr extends from approxi- 
mately nucleotide -3 to +5 . Within this region, nucleotide +2 appears to be unnecessary because complete Inr function is found with a T, G (mutant C4), or C (AdML Inr; ref. 15) at this position. It is noteworthy that the inactive mutant $\mathrm{Cl}$ contains the start site sequence for the TATA-containing human immunodeficiency virus type 1 (HIV-1) gene, which exhibits a 5 of 8 nucleotide homology with the TdT Inr (nucleotides -3 to +5 ). Although the HIV start-site region appears nonfunctional in our assay, previous mutagenesis of the HIV promoter suggested a role for this region in HIV transcription (27). Further analysis will be required to determine whether this homology is fortuitous or whether this HIV Inr is functional only in the context of the specific array of HIV control elements that have been identified both immediately upstream and downstream of the transcription start site (27-29).

In beginning to define the proteins involved in Spl/Inr transcription, we have found a requirement for mammalian TFIID, which previously was defined as a crude protein fraction required for transcription initiation $(3,4)$ and containing a TATA-binding activity (5). The TFIID requirement in the absence of a TATA box is consistent with studies of the adenovirus IVa2 gene (30), although it is not yet clear what element replaces TATA in this adenovirus promoter. The activities in the TFIID fraction that stimulate both Sp1/ TATA and Sp1/Inr transcription are likely to be carried out by the same protein or protein complex because of two observations: $(i)$ the two activities copurify through three columns and, most importantly, across a gradient elution from a high-resolution FPLC Mono S column; and (ii) both activities are unusually heat sensitive. However, because the mammalian TFIID fraction has not been purified to homogeneity, we cannot rule out the possibility that this fraction contains two distinct, heat-sensitive proteins that stimulate TATA- or Inr-mediated transcription.

The observation that yeast TATA-binding protein cannot effectively substitute for mammalian TFIID may provide insight into the structure and function of mammalian TFIID. The clearest deficiency of the yeast protein is its inability to stimulate transcription mediated by an Inr, as is evident from experiments with plasmids VI (Sp1/Inr) and IV (TATA/Inr). If the same protein in the mammalian TFIID fraction stimulates transcription from both TATA- and Inr-containing plasmids, then yeast TFIID may contain domains that are significantly diverged from HeLa TFIID such that they cannot mediate interactions required for transcription from an Inr. Alternatively, the yeast protein may lack additional domains or subunits that are found in mammalian TFIID. We favor this second possibility because $(i)$ mammalian TFIID chromatographs as a much larger protein than the $27-\mathrm{kDa}$ yeast TFIID (31), and (ii) DNase I footprinting analysis with partially purified mammalian TFIID shows more extensive protection of some promoters (6) than does yeast TFIID. In fact, because HeLa TFIID has been shown to protect from DNase digestion the start-site region of the Inr-containing AdML promoter, but not the start-site regions for several Inr-lacking promoters (6), HeLa TFIID may contain the polypeptide that interacts with the Inr. We do not yet know whether this putative, additional subunit is absent in yeast or whether it simply does not copurify with the yeast TATA-binding protein.

While analyzing the protein requirements for Inr-mediated transcription, we found that yeast TFIID could not activate transcription of our control plasmid V, containing the 21-bp repeats $30 \mathrm{bp}$ upstream of a TATA box. This deficiency is difficult to interpret because the results vary depending on which plasmid template is used. Clearly, transcription from the adenovirus E1B promoter, containing a single Sp1 binding site 8 bp from the TATA box, responds well, although the $S p 1$ response is observed only at high template concentrations. Two differences between these two plasmids are $(i)$ the distances between the Sp1 site and TATA box, and (ii) the number of SP1 binding sites. Neither of these differences is important, however, because a promoter containing two consensus Sp1 binding sites 30 bp from the E1B TATA box also responds well to the Sp1 sites in the presence of yeast TFIID (M.C.S., R. Segal, and A.J.B., unpublished results). Further experiments are necessary to determine the relevant differences between the two templates and to explain the template concentration dependence of the E1B promoter response.

We are grateful to Frank Pugh and Robert Tjian for discussing results prior to their publication, to Qiang Zhou for important contributions, and to Donald Rio for the use of his FPLC apparatus. We also thank Doug Black, Paul Kaufman, Donald Rio, and Marilyn Smith for critical reading of the manuscript. This work was supported by postdoctoral fellowships from the Helen Hay Whitney Foundation (S.T.S.), Arthritis Foundation (S.T.S.), and American Cancer Society (M.C.S.; PF2715), and by grants from the National Cancer Institute (A.J.B.; CA41062 and CA25235), American Cancer Society (D.B.; IM355T), Public Health Service (D.B.; GM39458), and the DuPont Center for Molecular Genetics (D.B.)

1. Johnson, P. F. \& McKnight, S. L. (1989) Annu. Rev. Biochem. 58, 799-839.

2. Breathnach, R. \& Chambon, P. (1981) Annu. Rev. Biochem. 50, 349-383.

3. Matsui, T., Segall, J., Weil, P. A. \& Roeder, R. G. (1980) J. Biol. Chem. 255, 11992-11996.

4. Samuels, M., Fire, A. \& Sharp, P. A. (1982) J. Biol. Chem. 257, 14419-14427.

5. Sawadogo, M. \& Roeder, R. G. (1985) Cell 43, 165-175.

6. Nakajima, N., Horikoshi, M. \& Roeder, R. G. (1988) Mol. Cell. Biol. 8, 4028-4040.

7. Parker, C. S. \& Topol, J. (1984) Cell 36, 357-369.

8. Buratowski, S., Hahn, S., Sharp, P. A. \& Guarente, L. (1988) Nature (London) 334, 37-42.

9. Hahn, S., Buratowski, S., Sharp, P. A. \& Guarente, L. (1989) Cell 58, 1173-1181.

10. Eisenmann, D. M., Dollard, C. \& Winston, F. (1989) Cell 58, 1183-1191.

11. Schmidt, M. C., Kao, C. C., Pei, R. \& Berk, A. J. (1989) Proc. Natl. Acad. Sci. USA 86, 7785-7789.

12. Horikoshi, M., Wang, C. K., Fujii, H., Cromlish, J. A., Weil, P. A \& Roeder, R. G. (1989) Nature (London) 341, 299-303.

13. Cavallini, B., Faus, I., Matthes, H., Chipoulet, J. M., Winsor, B., Egly, J. M. \& Chambon, P. (1989) Proc. Natl. Acad. Sci. USA 86, 9803-9807.

14. Dahlberg, J. E. \& Lund, E. (1988) in Structure and Function of Major and Minor Small Nuclear Ribonucleoprotein Particles, ed. Birnstiel, M. L. (Springer, New York), pp. 38-70.

15. Smale, S. T. \& Baltimore, D. (1989) Cell 57, 103-113.

16. Grosschedl, R. \& Birnstiel, M. L. (1980) Proc. Natl. Acad. Sci. USA 77, 1432-1436.

17. Chen, W. \& Struhl, K. (1985) EMBO J. 4, 3273-3280.

18. Maniatis, T., Fritsch, E. F. \& Sambrook, J. (1982) Molecular Cloning: A Laboratory Manual (Cold Spring Harbor Lab., Cold Spring Harbor, NY).

19. Dignam, J. D., Lebovitz, R. M. \& Roeder, R. G. (1983) Nucleic Acids Res. 11, 1475-1489.

20. Briggs, M. R., Kadonaga, J. T., Bell, S. P. \& Tjian, R. (1986) Science 234, 47-52.

21. Schmidt, M. C., Zhou, Q. \& Berk, A. J. (1989) Mol. Cell. Biol. 9, 3299-3307.

22. Jones, K. A., Yamamoto, K. R. \& Tjian, R. (1985) Cell 42, 559-572.

23. McKnight, S. L. \& Kingsbury, R. (1982) Science 217, 316-324.

24. Dynan, W. S. \& Tjian, R. (1983) Cell 35, 79-87.

25. Corden, J., Wasylyk, B., Buchwalder, A., Sassone-Corsi, P., Kedinger, C. \& Chambon, P. (1980) Science 209, 1405-1414.

26. Benoist, C. \& Chambon, P. (1981) Nature (London) 290, 304-310.

27. Jones, K. A., Luciw, P. A. \& Duchange, N. (1988) Genes Dev. 2, 1101-1114.

28. Garcia, J. A., Harrich, D., Soultanakis, E., Wu, F., Mitsuyasu, R. \& Gaynor, R. B. (1989) EMBO J. 8, 765-778.

29. Bielinska, A., Krasnow, S. \& Nabel, G. J. (1989) J. Virol. 63, 4097-4100.

30. Carcamo, J., Lobos, S., Merino, A., Buckbinder, L., Weinmann, R., Natarajan, V. \& Reinberg, D. (1989) J. Biol. Chem. 264, 7704-7714.

31. Reinberg, D., Horikoshi, M. \& Roeder, R. G. (1987) J. Biol. Chem. 262, 3322-3330. 